Activités

2-1 | avril 2005

Varia

\title{
Prévenir les TMS
}

De la biomécanique à la revalorisation du travail, l'analyse du geste dans toutes ses dimensions

From biomechanics to work valorization, analysing gesture in all dimensions

\section{Fabrice Bourgeois et François Hubault}

\section{(2) OpenEdition}

12 Journals

Édition électronique

URL : http://journals.openedition.org/activites/1561

DOI : 10.4000/activites. 1561

ISSN : $1765-2723$

Éditeur

ARPACT - Association Recherches et Pratiques sur les ACTivités

Référence électronique

Fabrice Bourgeois et François Hubault, «Prévenir les TMS », Activités [En ligne], 2-1 | avril 2005, mis en ligne le 02 avril 2005, consulté le 30 avril 2019. URL : http://journals.openedition.org/activites/1561 ; DOl : 10.4000/activites. 1561

\section{(a) $\odot \Theta$}

Activités est mis à disposition selon les termes de la licence Creative Commons Attribution - Pas d'Utilisation Commerciale - Pas de Modification 4.0 International. 


\title{
Prévenir les TMS \\ De la biomécanique à la revalorisation du travail, l'analyse du geste dans toutes ses dimensions
}

\author{
Fabrice Bourgeois \\ Ergonome consultant, omnia intervention ergonomique \\ omnia.f.bourgeois@nnx.com \\ François Hubault \\ CEP Ergonomie et Ecologie Humaine, Paris 1 Panthéon Sorbonne \\ ATEMIS \\ francois.hubault@wanadoo.fr
}

\begin{abstract}
From biomechanics to work valorization, analysing gesture in all dimensions

The outburst of musculoskeletal disorders in the early 1990's obliged ergonomics to rethink the way that health concerns and workplace organisation relate with each other. This is not to say that a purely biomechanical approach has become obsolete, however, efficient prevention strategies now require that management become the focal point in an ergonomic intervention, and not just one element of the global context. Ergonomic experience, particularly that which is oriented toward musculoskeletal disorder prevention, has progressively shown the pertinence of linking Tayloristic style management, still predominant in certain organisations, with pathological-based models for musculoskeletal problems in the workplace. Therefore one of the leading stakes in current ergonomics is to be able to put into action the activity model. This model permits us to connect prevention oriented models with models of economic and subjective work valorization. A preliminary step however, is for ergonomics to revalue it's own viewpoint of what it considers as activity.
\end{abstract}

\section{KEYWORDS}

Musculoskeletal disorders, activity, productivity (efficiency) performancee

\section{1.- Prévenir les TMS : où faut-il être ?}

Les TMS posent un problème particulier aux ergonomes. Tout le monde convient qu'il n'est pas suffisant de s'y prendre comme s'il s'agissait d'une entrée ordinaire de santé au travail. Les TMS sont identifiés comme complexes, au point que certains ont pu douter, ou doutent encore, que l'ergonomie ait vraiment la capacité d'intervenir efficacement en la manière. Pourtant, ce sont des maladies professionnelles, avec des facteurs de risque liés à des expositions professionnelles.

\section{1.- Le dilemme posé à l'ergonomie}

La difficulté s'illustre dans le dilemme bien connu en ergonomie de l'emploi ou la santé que les dirigeants d'entreprise, mais aussi les salariés, objectent, le plus souvent implicitement, à l'ergonome. Mais, il nous semble que plus qu'avant, il est suggéré que les TMS sont le prix de la santé à payer pour conserver-améliorer la compétitivité de l'entreprise et ... éviter-repousser des (nouveaux) plans sociaux, les délocalisations, etc. 
Que répondre ? Comment sortir de cette impasse dont le sentiment de fatalité finit par s'imposer jusque dans nos manières d'agir?

Trois scénarios sont possibles, entre lesquels il nous faut bien choisir.

Le premier choisit d'aménager les contraintes que les opérateurs supportent et dont ils subissent les conséquences (gênes, restrictions médicales, maintien à l'emploi menacé, exclusion ...). L'ergonomie se limite ici à l'amélioration du dimensionnement biomécanique du poste et l'humanisation des standards tayloriens. C'est ce que nous avons tous fait dans les années 90 en espérant que c'était là, au moins, un point de départ.

Le deuxième scénario choisit de renoncer. Les TMS sont vus comme des pathologies de la mondialisation, auxquelles on ne peut apporter aucun remède à l'échelle d'une entreprise. Un effet pervers de cette attitude est qu'en abandonnant de fait, alors, le champ de la transformation aux adeptes du premier scénario, les tenants de l'abandon participent eux-mêmes à la déconsidération de l'ergonomie qui ne se donne plus à voir que comme une technique d'aménagement de la tâche, un baume, ... un emplâtre sur une jambe de bois.

Le troisième scénario choisit d'aller voir de plus près si les TMS sont vraiment le coût que la société et les individus doivent supporter pour sauver des entreprises et des emplois. Ce scénario procède évidemment d'abord d'une conviction, mais aussi d'un savoir. Car, en effet, tout se joue, nous semble-t-il, dans la capacité à comprendre comment les tensions entre les exigences de la gestion et celles du travail peuvent générer aussi des ressources. Ce scénario, qui réclame des connaissances particulières est, pour nous, le seul à ouvrir de véritables perspectives d'intervention pour les ergonomes et, par voie de conséquence, de changement pour les salariés et les dirigeants. Mais il engage l'ergonome dans sa compétence (en objectivant les leviers de transformation et en sachant les mobiliser) et dans sa responsabilité (en explicitant les savoirs nécessaires aux conditions de réussite et en s'assurant de leur réalité).

\section{2.- L'espace épistémologique pour sortir du dilemme}

Situées pour la plupart dans le champ de la santé et de la conception, les demandes adressées aux ergonomes finissent par dresser un périmètre implicite de leur champ d'intervention qu'ils s'accordent d'ailleurs en général à ne pas dépasser. De fait, l'arbitrage final relève du dirigeant. Mais la délimitation du périmètre d'intervention est aussi induite par notre approche. Ainsi, alors que les processus de décision sont partie intégrante de leur domaine d'analyse, les ergonomes semblent plus discrets quand vient la question de la décision relative à la mise en œuvre de leurs propositions. Dès que l'affaire passe au stade de la mise en perspective économique, elle leur échappe. Le destin de leurs propositions n'est plus de leur ressort, et il peut prendre, de fait, toutes les formes : application, modification, atténuation, détournement à d'autres fins... ou le rejet.

Aussi, sur la durée, la transformation concrète des situations de travail reste-t-elle fragile. Discutée largement lors du séminaire de l'ANACT sur la Prévention durable (2004), cette fragilité fait écho à une ambiguïté : qu'entend-on par « apport ergonomique »? Très clairement, les salariés et les dirigeants attendent des solutions, c'est-à-dire des formules qui règlent le problème sur la base d'une maîtrise des déterminants et le contrôle des processus ... alors que, précisément en matière de TMS, il nous paraît que seules des réponses sont possibles, et qu'il ne faut donc pas trop s'étonner de voir le problème revenir dès lors qu'il n'avait en fait jamais vraiment pu disparaître (Hubault, 2005). Mais comment nos partenaires pourraient-ils alors se repérer dans l'univers étrange des « réponses »? On attend des solutions qu'elles soient « durables » pourvu que certaines conditions de pérennité, elles-mêmes maîtrisables et contrôlables, soient réunies. Mais que peut vouloir dire que des réponses durent, puisqu'elles sont par nature, imparfaites et provisoires ? Et le malaise 
s'accroît encore si on ajoute qu'en exigeant une solution, les entreprises attendent du consultant qu'il s'engage sur des résultats non seulement durables, mais aussi immédiats.

La difficulté tient, en fait, l'impossibilité pour la proposition ergonomique de s'intégrer au mode gestionnaire en place dans l'entreprise sans le changer. De fait, si la prévention des TMS requiert de faire évoluer le mode de gestion, il faut que l'intervention à la fois se coule dans le dispositif gestionnaire en place et l'infléchisse. En d'autres termes, qu'elle travaille dans les paradoxes du système, ses tensions internes... Prenons l'exemple très fréquent du stock intermédiaire entre les postes sur les lignes d'assemblage. La raison qui pousse à le limiter procède d'une «manière de voir » le processus productif qui ignore complètement la forme et la part que l'activité de travail prend dans la performance réelle. Selon ce modèle, la suppression ou la limitation des stocks induit immédiatement et durablement des gains de productivité et améliore immédiatement et durablement les indicateurs de performance. Et comme il assiste un mode de gestion de la qualité (les encours sont considérés comme des sources possibles de défauts de pièces et des immobilisations improductives), le modèle ne laisse aucune chance à la « manière de voir » de l'ergonomie, pour qui un stock intermédiaire peut constituer une ressource de régulation dans un système non stabilisé. En toute rigueur, il y aurait là, en fait, matière à rencontre : dans le modèle gestionnaire originel, l'existence d'un stock est un révélateur, un indicateur de tension, de «résistance» du réel; il vaut donc comme symptôme d'un problème qu'il désigne pour qu'on le traite. Ce n'est pas une position si éloignée de l'ergonomie pour qui la constitution de stocks intermédiaires indique, à travers la ressource qu'elle rassemble pour y répondre, l'existence de tensions opératoires. Mais la position gestionnaire théorique a glissé dans l'usage vers une pratique qui consiste à traiter le symptôme plutôt que le problème. Et c'est ce glissement, qui empêche la « rencontre », qu'il faudrait peut-être précisément essayer de renouer. L'opposition ne portera non plus alors sur la légitimité en soi des stocks, mais sur leurs raisons, et donc la nature du réel qui se trouve à l'œuvre dans « ce qui résiste ».

Or, face à ces modèles gestionnaires, les ergonomes abandonnent la partie trop tôt. Le risque d'instrumentalisation est alors évident, mais il dépend d'abord de nous de le contenir. Comment l'ergonome pourrait-il se soustraire à la question de savoir dans quel système de valorisation économique du travail ses recommandations ou prescriptions sont attendues ? Mais il est vrai qu'il lui faudrait d'abord le comprendre.

\section{2.- La multicausalité des TMS : quelle pluridisciplinarité ?}

Les ergonomes ont réagi dès que l'augmentation des déclarations de maladies professionnelles TMS s'est faite sentir, à la fin des années 80. Dès cette date, Maline (1991) faisait l'hypothèse d'un lien entre l'évolution du travail (notamment l'introduction du juste à temps ${ }^{1}$ et la mécanisation ou automatisation...) et l'évolution des contraintes gestuelles. Mais sur le terrain, cette hypothèse passait mal. Il fallait pour l'étoffer, des investigations plus précises des évolutions du travail identifiées comme contemporaines de la montée de cette pathologie. Et il restait à démontrer l'impact de ces évolutions sur les équilibres élaborés par les salariés avant ... qu'ils ne soient victimes de TMS. Cette démonstration a été laborieuse. La place du geste dans la compréhension de la survenue des TMS a eu du mal à s'imposer, comme si le geste concentrait sur lui les tensions et les contradictions de la pluridisciplinarité ergonomique.

1. «...Plusieurs interventions ... nous conduisent à penser que l'augmentation des affections péri-articulaires touchant les membres supérieurs est liée à des évolutions de l'organisation du travail. Des médecins de travail disent retrouver très souvent dans la discussion avec leurs patients, la notion de changements qualitatif et/ou quantitatif dans l'activité de travail ...»; « ... Ce flou (des données explicatives) nécessitent de s'enquérir de faits, d'évènements ou de données qui sont susceptibles d'avoir historiquement structuré les modalités du travail... » (Maline, 1991). 
Disponible et abondante dès la fin des années 80 , la source documentaire des physiologistes, notamment scandinaves et anglo-saxons (Kuorinka, \& Forcier, 1995), privilégie la description biomécanique. Pourtant, sous la houlette d'Armstrong et al. (1993), ces mêmes chercheurs ont vite repéré le rôle des facteurs psycho-sociaux et organisationnels dans la survenue de cette pathologie (Figure 1).

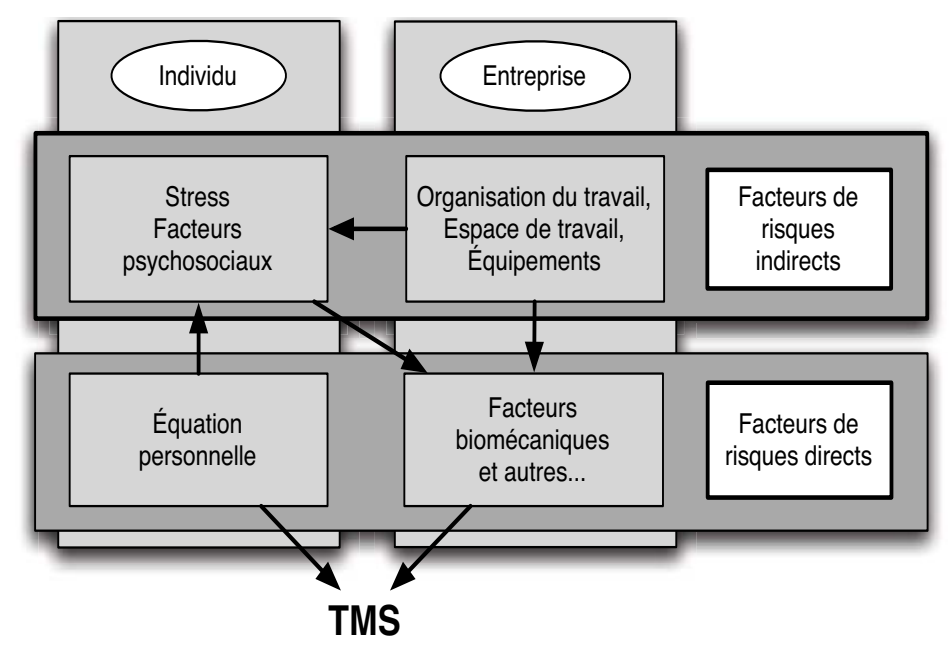

Figure 1.- Représentation consensuelle de la communauté scientifique, des facteurs de risque TMS - disponible dans les années 90 - source INRS (1996)

Malgré l'élargissement multi causal proposé, la contribution scientifique à l'explication des liens causaux est restée faible. De fait, le modèle de la figure 1 est comme embarrassé d'une distinction entre facteurs de risque directs et indirects. Elle invite à une relativisation des risques qui empêche de travailler cet élargissement... Ainsi, de fait, tout le monde ne peut qu'aller voir les facteurs dont « on est sûr que ... » et délaisser des facteurs « qui auraient avoir à voir avec mais comme on ne sait pas bien... ». On comprend l'écart qui s'ensuivra dans ces mêmes années entre les diagnostics ergonomiques capables tout de même d'élargir la compréhension, et la transformation réelle capable seulement de concerner ce qui est «direct » ou «sûr ». Par ailleurs, la catégorisation direct/indirect n'aide en rien pour éclaircir une autre discrimination, beaucoup plus « sensible », entre ce qui est imputable à l'individu (le sexe, l'âge, ses activités de loisirs, ...) et aux conditions du travail. Ainsi, en n'expliquant pas les liens, on accorderait aux facteurs biomécaniques d'être une question à adresser à l'entreprise. De même que le stress et les facteurs psychosociaux seraient en quelque sorte indissociés de l'individu. A la sortie, comme la distinction direct/indirect recouvre comme par hasard peu ou prou une distinction entre les facteurs ressortissant de modèles de sciences dures et ceux relevant de modèles de sciences molles, l'affaire est vite tranchée : tout se conclut, en pratique, en termes biomécaniques...

Si on soulage le modèle de cette double catégorisation (facteurs directs / indirects d'une part, et individu / entreprise, d'autre part), il gagne en pertinence, et nous pourrons alors renseigner chacun des termes et, surtout, leurs liens. Mais, il aura fallu ré-organiser, re-mobiliser et compléter les connaissances existantes. Sur les facteurs psychosociaux, les premières contributions ont été apportées par des enquêtes épidémiologiques ${ }^{2}$ tentant de montrer les liens entre le « vécu » des salariés et les contraintes de travail liées à l'organisation, les équipements, les types de gestes. La psychopathologie et la psychodynamique ont produit un matériau conséquent à la fin des années 90, pour comprendre les mécanismes de mobilisation psychique dans l'apparition des TMS (Pezé, 1999).

2. Enquête épidémiologique nationale Inserm-Anact-Inrs-Ccmsa-Dares 
A la fin des années 90, au moment du projet de rédaction de l'ouvrage de l'ANACT (Bourgeois, Lemarchand, Hubault, Brun, Polin, \& Faucheux, 2000), les auteurs étaient préoccupés par la difficulté de développer une véritable approche pluridisciplinaire de la prévention des TMS. C'est par la reprise de la question du geste que la piste s'est dégagée. Rétrospectivement cela apparaitt aujourd'hui évident, mais peut-être l'est-ce trop pour qu'on la voie. A l'époque, elle ne s'est pas imposée facilement.

\section{1.- La dimension cognitive : un oubli étonnant}

Le reproche que l'on peut faire au diagnostic biomécanique des sollicitations articulaires, c'est qu'il ne dit rien des intentions et des motifs qui sont à l'origine du geste. Ce dernier réalise une stratégie, en utilisant l'expérience des actions (dont des gestes) passées (Berthoz, 1997 et 98). Nous (re)découvrons là, l'activité cognitive qui fait du (des) geste(s) un processus permanent d'appréciation de la situation par l'opérateur et d'ajustement de ses modes opératoires. Le diagnostic des facteurs de risque TMS ne peut donc pas se contenter de décrire les entraves biomécaniques mais doit considérer aussi celles, cognitives, qui font obstacle à la recherche « des meilleurs gestes » pour l'opérateur.

Pour le peintre en bâtiment, écouter sa peinture « chanter » sur le mur c'est un moyen sûr, mêlé aux informations visuelles qu'il exploite aussi, pour corriger, ajuster, décider de la pression sur le rouleau, des directions des passes, de l'apport de diluant ... et ainsi économiser la peinture, son temps et ses gestes. C'est cette capacité de comprendre le chant de la peinture qui sous-tend la dynamique biomécanique des gestes. La figure 2, bien connu en ergonomie, illustre parfaitement comment le geste se maintient, non pas à l'identique bio-mécaniquement, mais à travers une recomposition finalisée. C'est, en quelque sorte, la capacité d'évaluation des résultats de ses propres gestes, par les sens - l'expérience - la mémoire - et le plaisir, qui détermine les gestes suivants.

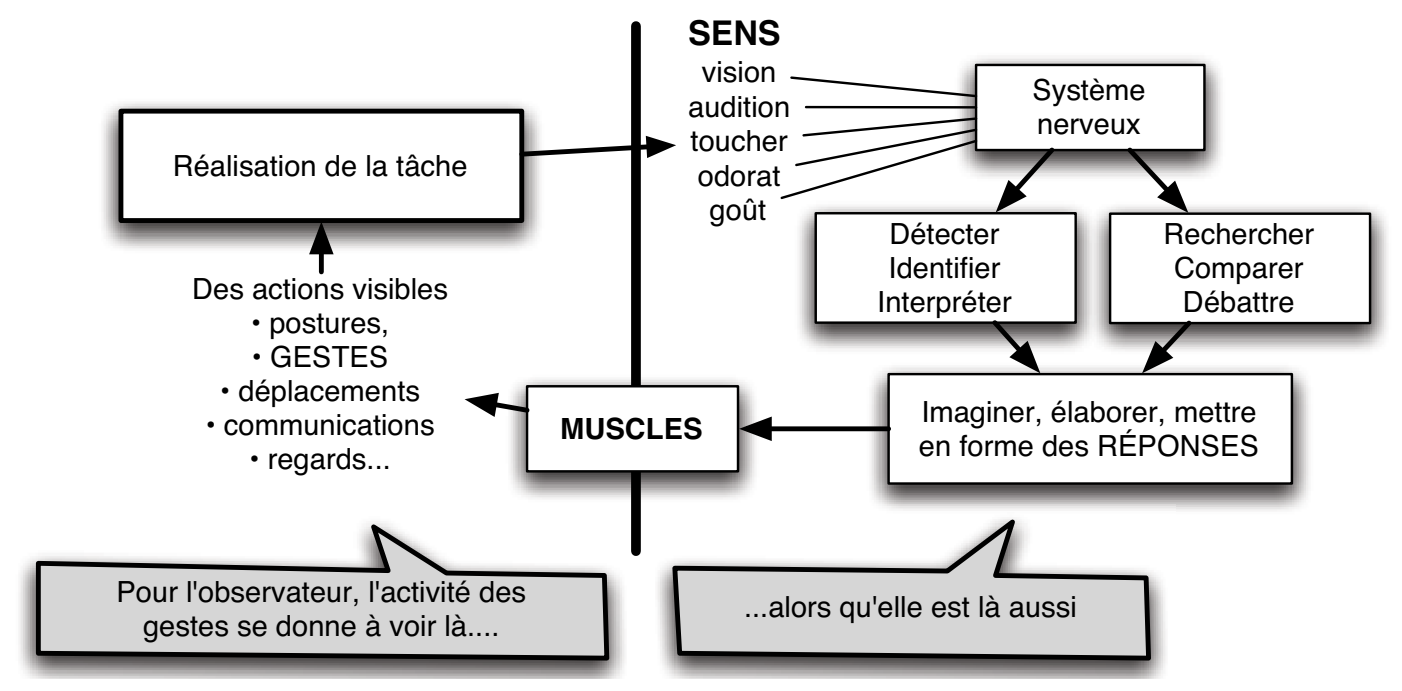

Figure 2.- Représentation des processus d'acquisition et d'interprétation des connaissances pour l'action et par l'action

Intégrer la dimension cognitive permet donc de comprendre que le peintre peut ne pas souffrir de TMS malgré son âge et son ancienneté : en gérant les conditions de la performance de ses gestes, il contribue à sa propre santé. Ainsi, pouvoir interpréter les évènements (dont les résultats de ses actions) et pouvoir y répondre de manière adaptée, déterminent les caractéristiques biomécaniques des gestes. L'analyse du geste par l'ergonome doit donc savoir identifier ces divers plans à partir des composantes biomécaniques observables des gestes (vitesse, direction, angle, répétitivité, force, ...). 


\section{2.- La dimension psychologique : un apport décisif}

Le geste a une fonction psychologique fondamentale. Quand les conditions sont réunies, le geste mobilise les ressources (ingéniosité, créativité, ...) qui permettent à la personne de faire face aux contraintes qu'elle affronte, et c'est ainsi qu'il participe de la construction de la santé mentale et physique. En revanche, si le geste est empêché (Clot, 1999), quand on ne parvient pas à faire le geste adéquat, le corps souffre. L'hyper agitation gestuelle peut alors constituer une stratégie défensive «pour tenir». Plus généralement, la sur-sollicitation de la voie gestuelle et l'accélération du geste représentent une « solution » psychosomatique possible pour accomplir un « geste vidé de sens » et pour masquer l'impossibilité de changer la situation.

S'il ignore cette dimension psychologique du geste, l'ergonome s'interdit toute compréhension du processus d'altération du geste. La prévention des TMS suppose un modèle d'analyse de l'activité qui intègre cette dimension psychologique du geste.

\section{3.- La dimension organisationnelle : le point de vue de l'activité}

Parmi les résultats de l'enquête INSERM-ANACT ${ }^{3}$, un facteur de risque TMS a retenu l'attention : la dépendance organisationnelle. Elle concerne notamment les possibilités de choix des temps de pauses et la latitude de varier le rythme de travail. Les personnes qui déclarent subir une dépendance organisationnelle élevée ont une plus grande probabilité $(1,43)$ d'avoir un syndrome du canal carpien (SCC). L'importance de ce facteur est encore plus important $(3,56)$ si on compare avec les personnes à faible dépendance organisationnelle qui ne travaillent ni en juste à temps, ni en flux tendu.

Depuis ces quinze dernières années, les exigences du marché poussent les entreprises accepter des séries plus courtes, des changements plus fréquents de production, des commandes urgentes, des interruptions pour essais et nouveautés, des normes à respecter pour obtenir et renouveler des certifications, des modifications de cahier des charges de leurs clients, des pressions sur les prix, etc. La prescription de souplesse fait que l'initiative, autrefois objet de méfiance, devient une prescription de l'organisation (par exemple, la prescription d'autonomie dans les lots de production, dans la relation de service, ....). La prescription évolue ainsi vers l'auto prescription, à travers une manière de « réquisition de la subjectivité » que la vitesse d'exécution et la simplification des opérations traduisent concrètement en pressions biomécaniques, psychiques et mentales de plus en plus fortes (Clot, 1999).

Nombreux sont ceux qui espéraient que les changements techniques et organisationnels offriraient des solutions au travail pénible et aux atteintes à la santé. Si certaines pénibilités reculent, d'autres émergent (Cristofari, 2003) et le phénomène n'épargne aucune entreprise. La place des femmes et des hommes a considérablement évolué dans les entreprises. Mais celles-ci l'appréhendent souvent mal car elles ignorent ou sous-estiment les conditions nouvelles de la contribution de leurs salariés à la performance, et négligent de se pencher assez sur la définition des moyens nécessaires.

\section{4.- La dimension économique : la pièce manquante}

La productivité mesure la contribution d'une ressource à un résultat. La productivité du travail, en conséquence, évalue l'apport du travail à la performance. Autrement dit, il s'agit bien de la place du travail dans le processus de création de valeur, du moins, dans la représentation qui en est faite. Cette évaluation s'établit en effet dans le cadre d'un modèle, une «manière de voir » et de penser la performance articulée à une «manière de voir » le travail. C'est là la scène où se joue la politique de

3. «Affections périarticulaires des membres supérieurs et organisation du travail - résultats de l'enquête épidémiologique nationale » DMT 65 TF 63 INRS 1996 
l'entreprise, et où sont comptées les ressources qu'elle mobilise pour réaliser sa performance. Tout ergonome est donc confronté aux effets que l'outillage conceptuel en place pourra avoir sur les marges de manœuvre de son intervention et la performance de ses actions de prévention. Or, cet outillage est très largement taylorien.

Taylor considérait la performance des gestes uniquement en termes énergétiques. Son modèle du fonctionnement de l'homme s'est perfectionné quand on produisit des répertoires de mouvements calibrés par des vitesses, des accélérations, des angles dans l'espace. Ce fut là, notamment, l'œuvre des Gilbreth. Sur cette base, le concept d' « opération » a permis d'élaborer des modèles de valorisation économique du travail qui sont encore très largement à l'œuvre. En témoigne par exemple, le modèle de la productivité taylorienne qui valorise la combinaison de l'accélération de la vitesse d'exécution des « opérations » (les délais et les débits) et de leur simplification.

Un tel modèle conduit l'entreprise à augmenter la productivité par la réduction des coûts directs, et particulièrement la réduction des coûts du travail direct. Ce modèle de la performance conduit donc à la réduction du geste à un mouvement élémentaire réalisant une opération élémentaire, selon une acception décorporalisée, désubjectivée, et donc trop pauvre du geste. L'approche biomécanique se trouve alors forcément très convoitée.

Pour sortir de cette impasse, on a argumenté la nécessité, certes ambitieuse, de penser «une autre productivité du travail », non plus associée à la réalisation d'opérations, mais à l'activité qui permet de tenir ensemble des exigences complexes et de faire face à des évènements (Bourgeois et al., 2000). Pour ce faire, il faut, d'une part, s'accorder sur une conception «plastique » du système de travail (Béguin, 2005), et, d'autre part s'accorder sur la nécessité d'identifier et de valoriser les « effets utiles » de l'activité humaine dans les systèmes événementiels (Hubault, 1999).

\section{3.- Un nouveau rendez-vous disciplinaire?}

Les conditions à réunir pour un tel projet concernent, incontestablement, les modèles de valorisation économique du travail. Penser le geste comme une activité, en termes d'activité, c'est une condition nécessaire pour reconsidérer sa contribution à la performance et ménager les moyens de la prévention des TMS. Puisque le geste demande des marges de manœuvre et réalise des régulations que l'opérateur juge utiles, alors la présence de TMS révèle un manque de souplesse du système qui empêche l'opérateur de faire face, par ses gestes, à la diversité et à la complexité des situations. Dès lors, la prévention des TMS consiste à décliner cette «manière de voir » dans la rigidité des systèmes de travail les raisons principales du phénomène, et à promouvoir une organisation du travail qui non seulement ne s'oppose pas mais pousse à développer les réponses de l'opérateur.

La clarification de la relation du geste avec l'organisation est donc décisive. De fait, les travaux récents démontrent l'intérêt d'aborder les TMS non plus seulement comme un problème de santé mais aussi comme une question industrielle. Mais pour creuser cette piste, il faut régler des rendezvous disciplinaires nouveaux, notamment avec l'économie et la gestion.

Les analyses du travail menées dans les années 70 (Laville, Teiger, \& Duraffourg, 1972) dans les productions de masse dressaient un tableau paradoxal. D'un côté, une façon pour l'opérateur pour « compenser » l'empêchement d'agir, consistait à mettre son « travail » à distance de lui-même, par une manière de s'impliquer que les sociologues ont appelé la « rêverie des OS », les médecins du travail parlant plus volontiers de schizophrénie... Une condition était nécessaire pour que cette rêverie et ces mouvements routinisés (ce qui ne veut pas dire automatisés) assurent quand même la commande sans que l'opérateur soit présent à ce qu'il fait : la standardisation de la production et sa stabilité dans le temps. La mobilisation répétitive de gestes réduits à des mouvements routiniers pour sortir des produits standards avec des équipements stabilisés, il fallait bien la conjonction de toutes ces conditions pour permettre l'évasion mentale grâce à laquelle l'opérateur assurait une pré- 
sence apparente. En grossissant le trait, la schizophrénie était pour ainsi dire les TMS de l'époque : l'indice de la pauvreté morale et mentale du travail. Ce n'était pour autant pas la seule réponse, et nous en connaissons d'autres qui permirent aux opérateurs de tenir un emploi trop pauvre en sollicitations : la flânerie, l'absentéisme, voire le «sabotage », sont autant de «comportements » dénoncés comme improductifs par l'organisation taylorienne alors qu'ils représentaient des stratégies « opératoires » pour tenir. D’un autre côté, les études ergonomiques menées dans les années 70 nous apprenaient que dans le monde taylorien réel, tout n'était pas aussi réglé que prévu, et que les évènements qui s'y produisaient quand même constituaient une ressource inconnue de l'organisation mais essentielle pour nourrir la présence des opérateurs, tant dans ses exigences de santé que d'efficacité (on a parlé des OS comme les « clandestins de l'efficacité »).

Ce que nous savons aujourd'hui, c'est que cette évasion ouvrière n'est plus possible et que le rappel à la vigilance a pris des proportions incomparables. L'éruption massive des TMS signe le recul des possibilités que représentaient ces « solutions ». Les raisons ont à voir, principalement, avec la gestion de la production : l'exigence économique croissante de variété et de variation interdit maintenant à l'opérateur toute distance avec son poste, même par l'esprit, même par la conversation, car les changements incessants réquisitionnent à tout instant son attention sans offrir pour autant plus de latitude, de marge, ni de sens, dans les gestes qu'il doit faire. Le travail est certes devenu moins monotone puisque les tâches sont plus variées, mais la répétitivité n'a pas diminué, on peut même dire qu'elle s'est multipliée en autant de fois que le changement (de gamme, d'outil, de lot, ...) oblige l'opérateur à s'adapter dans un mode opératoire qu'il lui est devenu plus difficile de construire et de maintenir. L'intensité s'est donc accrue. Et c'est cette tension requise au nom de la souplesse exigée par les conditions économiques nouvelles, que l'opérateur paye en rigidification articulaire.

La flexibilité réclame de l'opérateur une réactivité quasi réflexe et constante à des séquences qui changent souvent et soudainement. Mais cette réactivité demanderait que l'opérateur ait pu élaborer un geste assez sûr et puisse l'engager sûrement. Or c'est cette condition qui manque, justement : la possibilité pour un opérateur de tirer profit, de rentabiliser ses efforts pour acquérir des gestes efficaces, précis et économes pour sa santé ... . Cette possibilité est contrariée voire anéantie par le démarrage d'un nouveau produit, d'une nouvelle référence, d'une nouvelle série.... Quand le geste n'a pas d'autre perspective que sa réitération à l'infini, « faire le geste» ne délivre d'aucune tension, au contraire ça en crée (Jouanneaux, 2004 ; Hubault, 2004). Et à force de tension, la rupture est là.

Pour rendre compte de la dynamique de l'effort nécessité par la construction et le développement des gestes qui assurent un niveau de performance jugé «bon » par l'opérateur, l'analyse ne peut pas être seulement biomécanique. L'opérateur vise un palier de performance où les gestes ne font plus mal, où ils témoignent d'une habileté, et où ils produisent du plaisir en même temps que les résultats attendus. Pour cela, il faut satisfaire au moins les conditions suivantes :

- disposer d'assez de temps pour éprouver différentes façons de s'y prendre et trouver celle qui convient, à soi (âge, état de santé, expérience, ...) et à la situation (la tâche, les évènements, ...) ;

- éviter d'être dans l'urgence, auquel cas le palier de la performance est plus bas que prévu et les gestes réalisés plus dangereux pour la santé ;

- pouvoir profiter de ce palier pour récupérer des efforts qui ont été consentis pour y parvenir.

A chaque démarrage d'une nouvelle situation (démarrer une nouvelle série, redémarrer après une panne, intégrer un changement du cahier des charges qualité, travailler en binôme avec un nouveau, ...), un temps est nécessaire pour obtenir de ses gestes la performance souhaitée. Il faut essayer de nouveaux enchaînements opératoires, les corriger, les ajuster, s'y prendre autrement. ... Pendant ces temps de construction et de réajustement, les sollicitations sont très importantes, parfois nocives. S'il ne dispose pas du temps nécessaire pour (re)trouver ses paliers d'efficacité gestuelle avant qu'un nouveau changement l'oblige à chercher une autre configuration gestuelle, l'opérateur se 
retrouve en situation de cumul des sollicitations. Contraintes et astreintes augmentant de pair, il ne peut s'en sortir qu'en trouvant vite des modes plus économiques, sauf à se trouver précipité dans le risque de TMS, mais cela suppose une capacité qui se dégrade précisément quand il est victime de TMS...

Ce processus est trop souvent négligé dans la prévention. Nous avons longtemps mis monotonie et répétitivité à la même enseigne dans l'analyse du travail à la chaîne. Il est devenu impératif de distinguer ces notions car la répétitivité des gestes ne s'inscrit plus dans la monotonie. L'événement s'invite sans cesse dans le travail sans toujours l'enrichir pour autant. Les salariés sont aujourd'hui explicitement tenus d'assurer la continuité de la production $(0$ panne, 0 stock, 0 délai, 0 défaut, 0 accident,...) à travers une discontinuité grandissante des séquences (flexibilité-réativité-souplesse aux variations-variétés des commandes). Les opérateurs deviennent des transitaires d'une situation à l'autre et des « repreneurs en main » de ce qui risque à tout instant de déborder. Pour jouer ces rôles, il leur faut pouvoir retrouver ou inventer une bonne manière de s'y prendre. Or c'est là que le bât blesse : les difficultés propres à cette maîtrise sont rarement reconnues, ni donc la ressource opératoire requise en termes de marge de conduite.

Par sa construction multidisciplinaire, l'ergonomie est normalement bien équipée pour distinguer dans la démarche d'analyse du geste, ce qui est d'ordre stratégique pour l'opérateur (toujours lié à la singularité de la situation et à son dénouement) et ce qui est de l'ordre de la mise en œuvre de cette stratégie par lui, et que l'on peut décrire par ses caractéristiques biomécaniques.

\section{4.- L'ergonomie et la performance économique}

On l'a dit, l'ergonomie a bien rendez-vous avec la « performance économique ». C'est là que se joue sa rencontre avec l'approche gestionnaire des entreprises. Reste à définir ce terme de performance du point de vue ergonomique, et donc précisément sa relation avec l'activité de travail.

Le Larousse donne plusieurs acceptions à la performance. Les unes évoquent l'exploit (« le résultat obtenu par un athlète ou un cheval de course »), la réussite remarquable («faire si vite un travail »), les possibilités optimales d'un matériel. Pour les autres, plus sobres et proches de l'étymologie du mot (accomplir), il s'agit tout autant du résultat obtenu dans l'exécution d'une tâche que du mode qui le produit, particulièrement dans le cas des expressions artistiques où la performance consiste à produire un événement dont le déroulement constitue l'œuvre. Le terme a donc deux faces : résultat (état) et/ou déroulement (processus), qui font d'ailleurs écho à deux dimensions classiquement mobilisées dans l'usage gestionnaire du terme : la performance juge l'efficacité (degré de réalisation d'un objectif : mesure d'un résultat) mais aussi l'efficience (degré d'engagement d'une ressource dans ce résultat : mesure de l'intensité des efforts consentis pour l'obtenir). Sans précaution, l'usage du mot est donc périlleux.

Le registre de la performance humaine du travail (qui est plutôt un processus) est nécessairement différent du registre de la performance économique (qui est plutôt un résultat). Par ailleurs, les finalités (et donc l'appréciation de la réalité des «effets utiles») comme les efforts consentis (les « coûts ») n'ont aucune raison de s'apprécier à la même aune pour l'entreprise et pour les personnes. La « valeur du travail », a fortiori le jugement porté sur sa «productivité », n'a donc aucune raison d'être la même pour les deux «protagonistes ». L'analyse du travail a précisément pour mission d'instruire la «résonance » entre ces registres épistémologiquement et socialement disjoints (Hubault, 1996).

Dans l'ouvrage «Comprendre le travail pour le transformer» (Guérin, Laville, Daniellou, Durafourg, \& Kerguelen, 1997), la performance de l'opérateur est définie comme sa capacité à élaborer des modes opératoires, capacité qui résulte de ses tentatives de transformer une situation de contraintes en situation moins contraignante pour lui. C'est ce processus que décrit le modèle ergonomi- 
que classique de l'activité (Figure 3) qui souligne les conditions d'une contribution à la performance par l'activité.

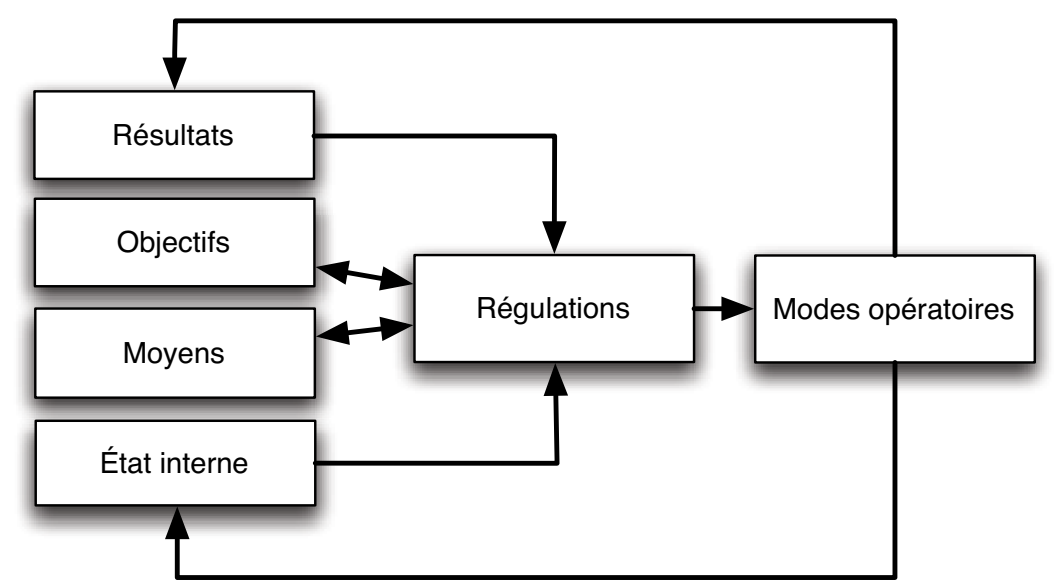

SITUATION "NON ASTREIGNANTE" : L'opérateur a des marges de manœuvre pour "déjouer" les contraintes en "agissant" sur les objectifs et les moyens

Figure 3.- Manière de représenter la performance humaine comme processus

Pour l'opérateur, travailler c'est pouvoir «maîtriser» les contraintes en «trouvant», élaborant, éprouvant des régulations et des modes opératoires adaptés. Vouloir transformer les situations de travail, tire sa légitimité de ce que l'opérateur doit pouvoir développer les initiatives qui lui permettent de «faire quelque chose » des contraintes qu'il rencontre, et qu'il est nécessaire pour cela d'agir à leur sources (au plan des causes ou des raisons qui se nouent dans les objectifs et/ou les moyens). « Déjouer les contraintes », c'est l'enjeu pour l'opérateur de se préserver de leurs effets délétères sur lui (santé) et sur les résultats économiques (efficacité). " Déjouer les contraintes », c'est également développer des compétences, prendre du plaisir (santé) en réalisant les résultats (efficacité). En d'autres termes, cela souligne que dans l'intervention ergonomique, l'analyse et la transformation ne doivent pas tant cibler les contraintes que les marges de manœuvres pour y faire face.

\section{1.- Les marges de manœuvre de l'opérateur peuvent-elles constituer des ressources?}

Il reste à savoir si le modèle de l'activité peut s'inscrire dans une projection économique de la transformation. Cette interrogation s'impose à quiconque prétend rejoindre, accompagner, rencontrer, aider ceux qui doivent, d'une manière ou d'une autre, fonder leurs choix sur des critères de performance (les dirigeants, les cadres d'Exploitation, RH, Méthodes, HSE, Qualité, les chefs de projet, les financiers, les contrôleurs de gestion, ...).

Pour rendre possible cette projection, une manière consiste à repérer les correspondances entre le modèle ergonomique de la performance et le modèle gestionnaire habituellement utilisé dans l'entreprise. Classiquement, la performance désigne pour les gestionnaires le système que composent ensemble le résultat, le mode engagé pour l'atteindre, et sa correspondance avec les objectifs visés (Figure 4). Elle met donc en jeu trois niveaux d'évaluation ${ }^{4}$ :

- l'efficacité ou la degré de réalisation des objectifs ;

- l'efficience ou le degré d'engagement des ressources pour produire les résultats ;

- la pertinence ou le degré de cohérence entre les objectifs et les ressources. 


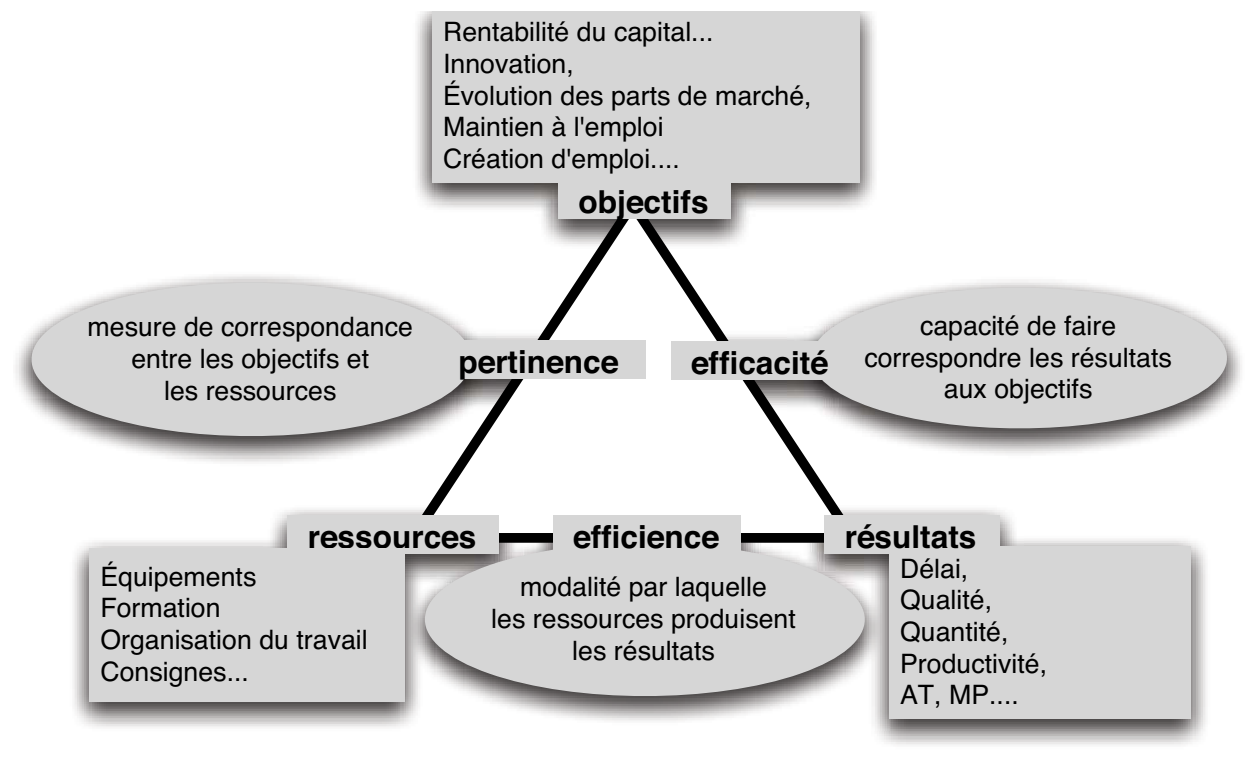

Figure 4.- Modèle « classique » du gestionnaire.

Les objectifs peuvent former un champ très large (innovation, évolution des parts de marché, rentabilité du capital, ...) dans lequel le dirigeant peut décider lui-même ou hériter d'une décision qu'il a charge d'appliquer. Cette diversité complique évidemment la mesure intrinsèque de chacun des objectifs dans sa déclinaison en termes de ressources et résultats. Au point que la performance finit par décrire davantage la stratégie, l'envie, l'aspiration à un but ou à un idéal du futur (objectifs) alors que sa mesure ne peut s'appuyer que sur le passé, au mieux le présent (moyens et résultats) (Lebas, 1996).

Les ressources désignent d'un coté l'ensemble des moyens prévus pour atteindre les objectifs, mais ce sont aussi les moyens réellement engagés pour réaliser un résultat. Les « ressources humaines » expriment ainsi le volume et la nature des contributions « humaines » attendues pour atteindre les objectifs, mais aussi le volume et la nature des contributions « humaines » réellement engagées pour réaliser les résultats... L'hésitation de la «pensée $\mathrm{RH}$ » sur ce qu'elle entend vraiment par « RH » (en termes d'effectifs, de qualifications, de contenus d'activité, d'organisation ?), et sur l'axe dans lequel elle l'instruit (en termes d'intention stratégique, en termes de réalisation opérationnelle ?) est un vrai handicap.

Les résultats sont illustrés par des mesures ou des indicateurs inspirés en grande partie par les besoins de la comptabilité analytique. Sur l'axe de l'efficience, la productivité délai/débit relève de modalités de calcul (le RGI ou le nombre d'heures théoriques d'ouverture possible des machines, le SET UP ou les temps de changements d'outillage et de références, l'efficience globale ou le ratio entre le nombre d'heures gammées et le nombre d'heures travaillées tout personnel, le WIP ou le temps de traversées d'un produit dans l'atelier, stocks matières premières, les rebuts, l'absentéisme,

4. Cette schématisation tend aujourd'hui à se complexifier dans la configuration immatérielle qui se développe dans toutes les organisations productives, y compris industrielles, du fait que la production (d'un bien ou d'un service) ne fait pas seulement que consommer de la ressource (enjeu d'efficience, de productivité), mais aussi en produit (enjeu d'efficacité « durable », de pérennisation de l'activité, de consolidation de la réputation... par l'apprentissage, la capitalisation des expériences, la consolidation des collectifs, la santé, l'implication, ...) ou en détruit (ce qui n'est pas équivalent à consommer). Cela change considérablement, alors, la manière même de « dimensionner » l'efficacité et la productivité... 
...) sur lesquelles il est impératif que les ergonomes développent une pensée s'ils ne veulent pas rester empêchés d'infléchir quoi que ce soit dans certains secteurs.

Le modèle qui soutient l'instrumentation gestionnaire n'est pas toujours en phase avec les formes de pensée ou «manières de voir » qui inspirent l'action des décideurs. Identifier ce paradoxe est déterminant pour comprendre les marges de manœuvre dans lesquelles les responsables se sentent pouvoir évoluer. Une ligne de front s'impose alors à l'ergonomie quand ses propres analyses viennent croiser ce paradoxe (Sablon, 2004).

Mais cette perspective n'est pas sans tensions. Elles concernent principalement la relation « sensible » entre productivité, qualité et rentabilité. Dans le modèle taylorien qui inspire la plupart des modes de gestion encore en place, la qualité est supposée stabilisée, de sorte que l'augmentation de la productivité est le moyen privilégié de la rentabilité. Les responsables opérationnels, eux, « savent» que la qualité réelle est beaucoup moins stable, a fortiori dans l'économie des services, et que l'augmentation de la productivité peut donc la dégrader et induire des effet négatifs en termes de rentabilité. Mais ils restent jugés sur les bases d'un reporting ${ }^{5}$ qui ignore ce processus, et par ailleurs ils peinent à recomposer un modèle cohérent opposable...

Pour l'ergonome, intervenir suppose donc de bien connaître la géographie de ces tensions, les failles dans lesquelles il doit savoir trouver les leviers pour accompagner les lignes de forces favorables à la reconnaissance de la valeur du travail.

La «performance » est un construit social qui dépend du modèle choisi pour la gérer. Ce n'est pas une « donnée ». Il y a place pour la discussion sur le périmètre et les modalités de son évaluation. Si les descripteurs n'intègrent ni les engagements ni les contributions des personnes, l'activité en somme ..., charge à nous que ça change.

La figure 5 met les modèles en résonance. Dans les correspondances qui les relient l'un à l'autre, elle tente de montrer comment les dimensions gestionnaires se retrouvent dans le modèle de l'activité. Bien sûr, des précautions doivent être prises dans la superposition, car on notera que :

- les objectifs de l'entreprise ne sont pas exactement les objectifs de la prescription du travail mais ils y sont de toutes façons ;

- les résultats de l'entreprise ne sont pas tous portés à la connaissance du salarié et, inversement, les résultats significatifs pour le salarié ne sont pas forcément connus par l'entreprise ;

- la fonction « ressources humaines » apparaît dans le modèle de l'activité, à la fois dans les moyens (coopérations, dimension collective de travail, ...) et dans l'état interne (état de santé, âge, formation, expérience, ...) ;

- l' «état interne» de l'engagement et de la contribution de la personne n'est pas connu, et rarement reconnu, comme ressource par l'entreprise.

5. système d'information remontant construit à partir d'indicateurs de résultats (délai, qualité, réactivité,...) 
Dans cet exercice d'incorporation d'un modèle dans l'autre, il faut faire apparaitre les régulations et les modes opératoires qui ne sont pas représentés au départ dans le modèle gestionnaire classique.

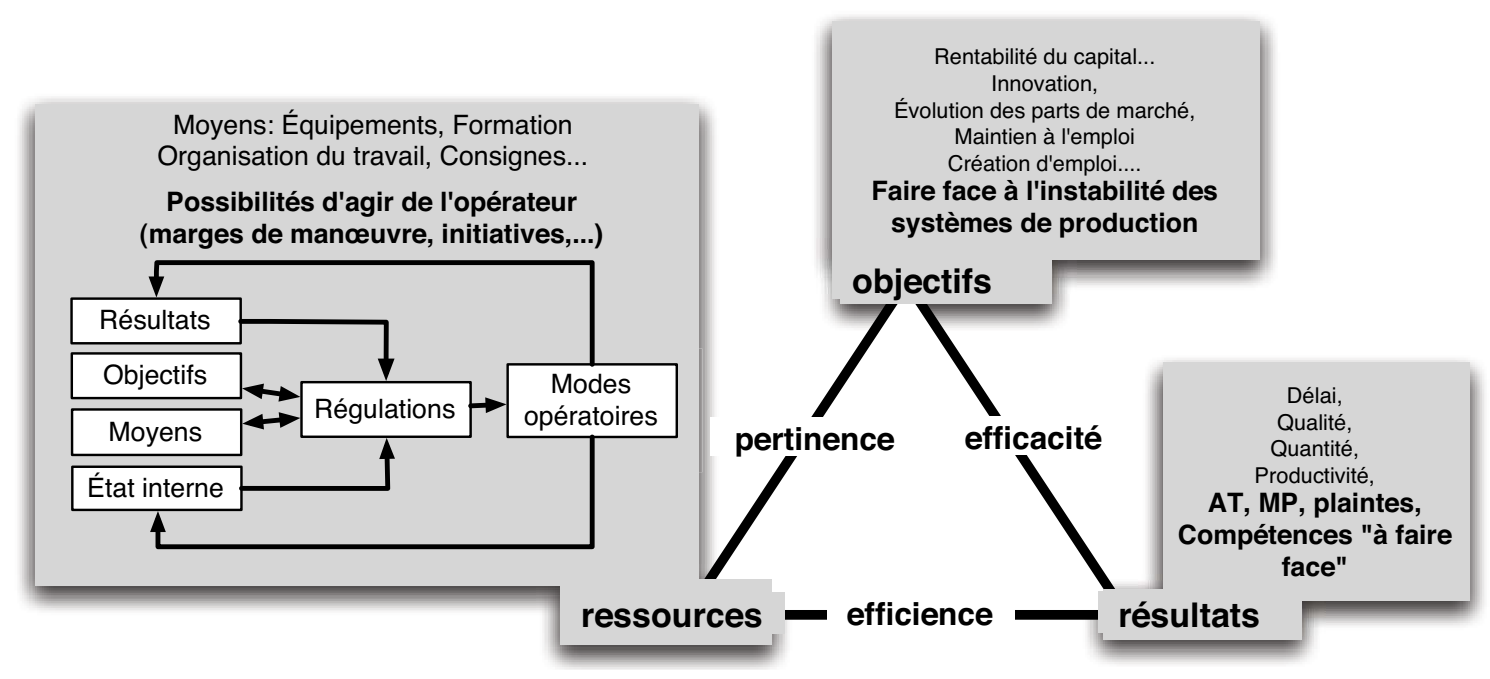

Figure 5.- Proposition de rencontre des modèles de performance

De fait, la rencontre de ces deux modèles discute la question de la valorisation du travail. Si par exemple, nous ajoutons au nombre des objectifs, la nécessité de faire face à l'instabilité des systèmes de production, cela appelle de pourvoir en ressources en conséquence, et incidemment de reconnaître l'importance de suivre l'évolution de l'état interne (en tant que ressource permettant de produire des régulations et des modes opératoires efficaces). Dans les résultats, des indicateurs d'efficience des régulations et des modes opératoires employés peuvent alors être pris en compte (cf. développement de compétences, plaintes TMS...).

\section{2.- L'analyse de l'activité peut-elle produire des indicateurs de performance?}

Dans la figure 6, la mise en exergue d'indicateurs d'activité et de résultats (Hubault, 1996) offre le possibilité d'aborder les distinctions établies en gestion entre pertinence, efficience et efficacité, dans les termes proposés par le modèle de l'activité de travail.

- le conflit de logiques qui naît de la rencontre entre la logique technico-organisationnelle (la tâche) et la logique du vivant (la personne, le travailleur, le salarié, dans leurs dimensions sociales, sociologiques, psychophysiologiques - l'état interne -, psychiques, ...), résonne avec la tension décrite entre objectifs et ressources en termes de pertinence ;

- le processus de définition des ressources à partir des objectifs ne peut esquiver la prise en compte de ce conflit de logiques. S'il fait l'impasse, il ruine son potentiel d'initiatives et de marges de manœuvre. A l'inverse, le prendre en compte, c'est le moyen de mettre en cohérence objectifs et ressources ;

- de même, la capacité de l'opérateur de trouver les compromis opératoires (régulations et modes opératoires) pour gérer ce conflit de logique, relève de l'efficience ; 
- les dimensions économiques pour l'entreprise et humaines pour l'opérateur de la performance du travail, renseignent enfin l'efficacité du système de travail.

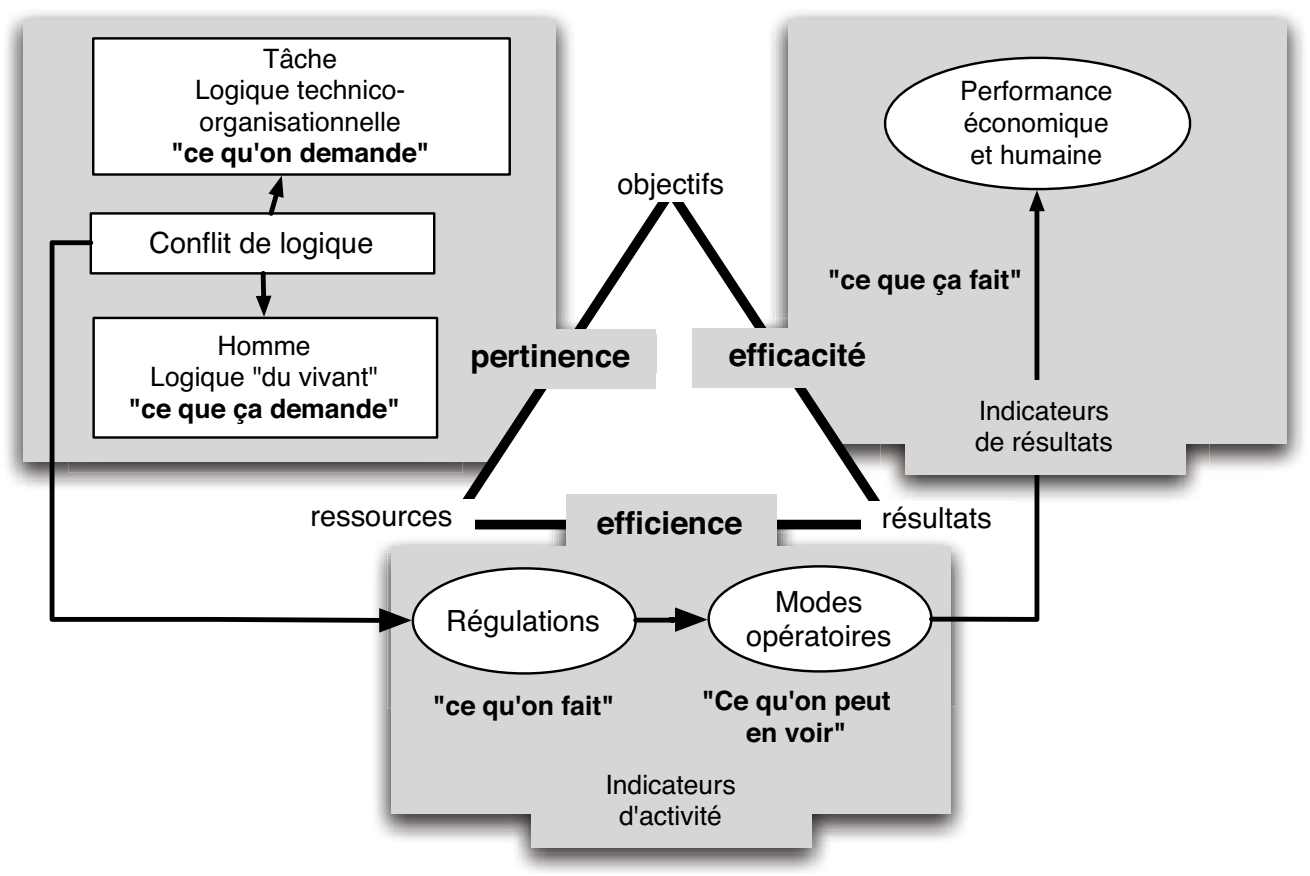

Figure 6.- L'analyse de l'activité (Hubault, Noulin, \& Rabit, 1996) instruisant la pertinence, l'efficience des modèles de performance.

\section{5.- Conclusions}

Il est évident que cette tentative d'interpellation entre modèles n'est pas sans risque.

J. Leplat et M. de Montmollin (2004) identifient les sciences de la gestion selon deux approches. L'une est développée en proximité avec la sociologie et les sciences dans des centres de recherche en gestion l'autre, animée disent-ils par une sorte de frénésie à l'égard des modèles étrangers, procède surtout de séduction. Aucune (mais surtout la seconde) n'a vraiment de valeur scientifique selon eux, et ils considèrent que l'ergonome se doit simplement de les connaître. Dans l'inventaire très détaillé des rapports que l'ergonomie entretient entre les connaissances et l'intervention, $\mathrm{C}$. Gadbois et J. Leplat (2004) ne font pas mention des connaissances économiques.

La question de la productivité est pourtant incontournable. L'usage économe de la ressource est au carrefour du sens du travail tant en termes subjectif (contribution/rétribution), social (utilité/disponibilité), qu'économique (principe d'économicité, effet utile/coût). Et il faut bien reconnaître que la productivité taylorienne résiste à tous les effets de modes, et contrecarre frontalement l'ambition de l'ergonomie qui s'y casse plus souvent les dents que l'inverse. L'avenir de l'ergonomie peut-il vraiment se penser à distance trop grande des questions économiques qu'elle rencontre et qu'elle pose ?

Cette conception n'est pas encore partagée. Des rendez-vous sont manqués, participant et précipitant des oppositions au sein même de la discipline. C'est particulièrement le cas dans la prévention des TMS.

Dans son analyse sur le nouveau productivisme, Ph. Askenasy (2004) laisse entendre que l'ergonomie est à l'origine de la spectaculaire régression des TMS aux Etats-Unis. Cette affirmation flatteuse laisserait profiler une évolution de même type en France (avec les 10 années régulières de retard par rapport aux Etats-Unis). Elle nous laisse cependant pantois tant les préconisations citées (formations 
des salariés et de l'encadrement aux bases de l'ergonomie et aux règles de sécurité, aux postures adéquates ...) nous semblent relever de modifications mineures et peu efficaces au vu de notre expérience des années 90 en France.

Les transformations doivent viser un registre plus ambitieux. Il s'agit de favoriser la plasticité des systèmes de travail, c'est-à-dire rendre possible le développement d'une variété de formes organisées, «trouvées » localement et collectivement, en rapport très singulier avec la situation, ellemême condamnée à être de plus en plus particulière, éphémère, complexe, ...

L'analyse ergonomique du geste permet de préciser les déterminants de cette plasticité nécessaire à l'efficacité de l'activité. L'indicateur de conception n'est plus le geste à faire, mais le geste possible, la possibilité de geste. Des exemples d'intervention illustrent la précision de la réponse que l'on peut apporter en conception. Plus d'une fois, il nous est arrivé de redonner sa valeur de ressource à un stock intermédiaire que la logique de gestion classique condamnait comme un coût. Cette modification du statut de l'encours constitue un véritable renversement du modèle d'efficacité, et cela n'a été à chaque fois possible que par l'effet d'un autre renversement, la reconnaissance du rôle déterminant de l'activité dans l'efficacité. D'autres exemples objectivant un seuil de fatigue dans telle configuration productive, ou l'effet finalement utile d'opérations jugées a priori comme non productives ... illustrent, de la même façon, la capacité des ergonomes à introduire de la plasticité dans la conception des systèmes de travail. Mais cette capacité restera limitée tant qu'elle ne sera pas "expliquée » avec les modèles de gestion qui dominent la pensée des ingénieurs et des managers de projets.

Il n'y pas trente six façons d'envisager cette confrontation. L'ergonome doit développer des connaissances alternatives pour la conception de systèmes de travail sur une base argumentative qui explique en quoi il est contre-productif de complètement pré-écrire l'acte de travail (Béguin, 2005). L'ergonomie joue là sa vocation de promouvoir le travail comme « une culture de la vigilance », « une culture de la présence » qui développent des compétences d'intervention, une «tolérance à l'événement ». (Bourgeois et al.,2000).

Il n'y a là rien pour effrayer spécialement nos interlocuteurs dans les entreprises. Mais comme aucun modèle économique de valorisation du travail, aucune approche de la productivité sensible à la culture de la vigilance, à l'efficacité de la plasticité, n'est disponible à leur niveau pour soutenir le développement d'autres modèles, ils ne sont pas disposés à emprunter des chemins encore mal balisés. Bref, le problème n'est pas l'absence d'alternatives, mais la capacité des ergonomes à renseigner le point de vue économique de la même façon qu'ils ont appris à renseigner de manière innovante, le point de vue de l'ingénierie, de l'architecture et de la technologie. Il nous faut apprendre à le faire.

\section{BIBLIOGRAPHIE}

Armstrong ; T.J., Buckle, P., Fine, L.J., Hagberg, M., Jonsson, B., Kilbom, A., et al. (1993). A conceptual model for work-related neck and upper limb musculoskeletal disorders. Scandinavian Journal of Work Environment \& Health, 19(2), 73-84

Askenasy, P. (2004). Les désordres du travail - enquête sur le nouveau productivisme, la république des Idées. Paris: Seuil.

Béguin, P. (2005). L’ergonomie en conception : Cristallisation, plasticité, développement. In B. Weil, \& A. Hatchuel (Eds). Les nouveaux régimes de la conception. (à paraître).

Berthoz, A. (1997). Le sens et le mouvement. Paris: Edition Odile Jacob - Sciences.

Berthoz A. (1998). Manifeste pour la réintégration de l'action du mouvement au cœur de l'étude du cerveau. Sciences et vie, hors série, $\mathrm{n}^{\circ} 204$, septembre.

Bourgeois, F., Lemarchand, C., Hubault, F., Brun, C., Polin, A., \& Faucheux JM. (2000). TMS et travail, quant la santé interroge l'organisation. Lyon: Editions de l'ANACT. 
Clot, Y. (1999). La fonction psychologique du travail. Paris: PUF.

Cristofari, M.F. (2003). Bilan des sources quantitatives dans le champ de la santé et de l'itinéraire professionnel. CEE 03/11 (disponible sur www.cee-recherche.fr)

Gadbois, C., \& Leplat, J. (2004). Connaissances et interventions. @ctivités, 1 (1) http://www.activites.org/ v1n1/html/gadbois.html

Guérin, F., Laville, A., Daniellou, F., Duraffourg, J., \& Kerguelen A. (1997). Comprendre le travail pour le transformer. La pratique de l'ergonomie. Lyon: Editions de l'ANACT

Hubault, F. (1996). De quoi l'ergonomie peut-elle faire l'analyse ? In F. Daniellou (Ed.), L'ergonomie en quête de ses principes - débats épistémologiques (pp. 103-140). Toulouse: Éditions Octarès.

Hubault, F. (1999). Engagement du travail, engagement dans le travail, les enjeux de la mesure de la productivité du travail. Performances Humaines et Techniques, $\mathrm{N}^{\circ}$ Hors Serie «Ergonomie et productivités, questions mutuelles ?, séminaire Paris1, », 2-13.

Hubault, F. (2004). La ressource du risque. In F. Hubault (Ed.), Travailler, une expérience quotidienne du risque?, Séminaire Paris 2003 (pp. 207-220). Toulouse: Editions Octarès.

Hubault, F. (2005). Activité, Travail, Ressource Humaine : parle-t-on de la même chose ? Les stratégies du changement en question. In Actes du séminaire Paris1, 1-3 juin 2004. Toulouse: Éditions Octarès (à paraître).

Hubault, F., Noulin, M., \& Rabit, M. (1996). L'analyse du travail en ergonomie. In P. Cazamian, F. Hubault, \& M. Noulin (Eds), Traité d'ergonomie (pp. 289-309). Toulouse: Editions Octarès.

INRS (1996). Les troubles musculosquelettiques du membre supérieur. La ligne prévention.

Jouanneaux, M. (2004). La vitalité au travail permet la maîtrise des situations à risque. In F. Hubault (Ed.), Travailler, une expérience quotidienne du risque?, Séminaire Paris 2003 (pp. 67-84). Toulouse: Editions Octarès.

Kuorinka, I., \& Forcier, L. (1995). LATR Les lésions attribuables au travail répétitif. Paris: Editions Maloine

Laville, A., Teiger, C., \& Duraffourg, J. (1972). Conséquences du travail répétitif sous cadence sur la santé des travailleurs et les accidents. Paris: Rapport n ${ }^{\circ} 29$, Laboratoire de physiologie du travail et d'ergonome CNAM.

Lebas, M (1996). Performance : mesure et management. Faire face à un paradoxe. In P. Cazamian, F. Hubault, \& M. Noulin (Eds), Traité d'ergonomie (pp. 547-572). Toulouse: éditions Octarès.

Leplat, J . , \& de Montmollin, M. (2004). Les voisinages disciplinaires de l'ergonomie. In P. Falzon (Ed.), Ergonomie (pp. 51-65). Paris: PUF.

Maline, J. (1991). Dossier affections péri-articulaires et organisation du travail. La lettre d'information de l'ANACT, n¹66, septembre.

Pezé, M. (1999). Les athlètes au quotidien : approche psychosomatique et psychodynamique des TMS. In F. Bourgeois (Ed.), TMS et évolution des conditions de travail. Actes du séminaire Paris 1998 (pp. 63-70). (téléchargeable sur le site www.anact.fr).

Sablon, S. (2004). La contribution de l'ergonome au fonctionnement de l'entreprise : la mise en ouvre de l'ARTT dans deux entreprises de construction. Thèse de doctorat d'ergonomie, Université Bordeaux 2.

\section{RÉFÉRENCEMENT}

Bourgeois, F., \& Hubault, F. (2005). Prévenir les TMS. De la biomécanique à la revalorisation du travail, l'analyse du geste dans toutes ses dimensions @ctivités, 2 (1), 19-36, http://www.activites.org/v2n1/ bourgeois.pdf

\section{RESUMEN}

La brutal irrupción de los TME ha obligado a la ergonomía, a partir de los años 90, a replantearse la relación entre salud y organización del trabajo. No es que el enfoque biomecánico haya perdido legitimidad, sino que la eficacia de la prevención exige un cambio en el campo de acción de la 
intervención que hace que se consideren a la gestión y al management ya no como elementos del contexto, sino como la materia misma de la intervención. La experiencia de la ergonomía en el campo de los TME ha ido cimentando el convencimiento de la pertinencia de relacionar el modelo patológico de los TME al modelo de gestión taylorista que, todavía, inspira a las organizaciones involucradas. En este sentido, el desafío de la ergonomía es de mobilizar el modelo de la actividad a fin de poder relacionar los modelos de prevención de riesgos con los de la valorización económica y subjetiva- del trabajo, pero a condición de que ésta realice un reposicionamiento de su propia comprensión de lo que denomina como " actividad "

PALABRAS CLAVE

TME, actividad, productividad, desempeño

\section{RÉSUMÉ}

L'irruption brutale des TMS a obligé l'ergonomie, dès les années 90, à repenser le rapport entre santé et organisation du travail. Non pas que, en l'occurrence, l'approche biomécanique ait perdu toute légitimité, mais parce que l'efficacité de la prévention demande un changement de périmètre de l'intervention qui fait de la gestion et du management, non plus des éléments de contexte, mais la matière même de l'intervention. L'expérience de l'ergonomie en matière de prévention des TMS, l'a en effet progressivement convaincue de la pertinence de relier le modèle pathologique des TMS au modèle de gestion taylorien qui inspire encore les organisations concernées. D'où l'enjeu pour l'ergonomie, de mobiliser le modèle de l'activité pour relier modèles de prévention des risques et modèles de valorisation - économique et subjective - du travail. A condition évidemment, de procéder elle-même à un repositionnement de sa propre compréhension de ce qu'elle appelle « activité ».

\section{MOTS CLÉ}

TMS, activité, productivité, performance 